\title{
Effect of transnasal insufflation on sleep-disordered breathing in acute stroke
}

\author{
José Haba-Rubio • Daniella Andries • \\ Vincianne Rey-Bataillard • Patrik Michel • Mehdi Tafti • \\ Raphael Heinzer
}

Received: 13 November 2012 / Accepted: 18 December 2012 /Published online: 28 October 2014

(C) Springer-Verlag Berlin Heidelberg 2012

We thank Drs Esquinas and Nilius for their interest in our article [1]. They suggest that transnasal insufflation (TNI) should not be used in severe OSA cases or in cases requiring intubation. We fully agree that the patient who can tolerate CPAP should use it, and we never proposed TNI as an alternative to intubation. Our results, however, suggest that TNI could be an alternative treatment to CPAP for sleep-disordered breathing (SDB) after a stroke.

Concerning their comments on our methodology, patients with severe neurologic deficits, including aphasia, were excluded in this preliminary study. Nevertheless, there are nonverbal ways to communicate with patients with aphasia in order to determine their tolerance to TNI (or other

J. Haba-Rubio $\cdot$ D. Andries $\cdot$ V. Rey-Bataillard $\cdot$ M. Tafti $\cdot$

R. Heinzer

Centre d'Investigation et de Recherche sur le Sommeil, Centre Hospitalier Universitaire Vaudois and Université de Lausanne, Lausanne, Switzerland

V. Rey-Bataillard $\cdot$ P. Michel

Service de Neurologie, Centre Hospitalier Universitaire Vaudois and Université de Lausanne, Lausanne, Switzerland

M. Tafti

Centre Intégratif de Génomique, Centre Hospitalier Universitaire

Vaudois and Université de Lausanne, Lausanne, Switzerland

J. Haba-Rubio $(\bowtie)$

Center for Investigation and Research in Sleep (CIRS), Lausanne University Hospital (CHUV), 1011, Lausanne, Switzerland

e-mail: Jose.haba-rubio@chuv.ch treatment), and we believe that even aphasic patients could be included in a further protocol on TNI.

We agree that it would have been interesting to have sleep studies prior to the stroke in these patients, but obviously, this could not be done in this protocol since patients were recruited after the stroke. Moreover, the objective of our study was not to determine the effect of stroke on SDB. We agree that a randomized study against CPAP would be interesting, but the aim of the present study was only to determine if TNI could be a usable treatment alternative in the acute phase of stroke.

As mentioned in the discussion of our article, we used a fixed and relatively low flow rate of $18 \mathrm{~L} / \mathrm{min}$ to guarantee a maximum of comfort with the TNI, but it is possible that with a higher flow rate, a further reduction of SDB could have been achieved.

Finally, we agree with Drs Esquinas and Nilius that further studies with a clinical follow-up are necessary to determine if treatment with TNI in the acute phase of stroke can improve the long-term outcome of these patients.

Conflict of interest There is no conflict of interest to disclose.

\section{Reference}

1. Haba-Rubio J, Andries D, Rey V, Michel P, Tafti M, Heinzer R (2012) Effect of transnasal insufflation on sleep disordered breathing in acute stroke: a preliminary study. Sleep Breath 16:759-764 